\title{
Analyse comparative des méthodes de calcul des pieux forés isolés à partir de l'essai SPT
}

A. BOUAFIA

Maitre de conférences à l'Université de Blida Alqérie

48, rue Hassiba-Ben-Bouali

16016 Alger, Algérie bouafia.ali@caramail.com
La littérature géotechnique est assez riche en méthodes de calcul de la capacité portante des pieux isolés forés dans le sable à partir de l'essai de pénétration standarc SPT. Ces méthodes ont été essentiellement développées à partir de l'analyse empirique des essais de chargement statique vertical des pieux. Les prévisions de ces méthodes sont souvent contradictoires et rares sont les études comparatives pour l'évaluation de la qualité cle prévision de ces méthodes à partir des expériences de chargement des pieux.

L'article se propose de contribuer à l'évaluation de quelques méthodes couramment utilisées en pratique, à travers une banque de données d'essais de pieux forés dans des sites sableux, en comparant les valeurs prédites de la charge verticale limite à celles interprétées de l'essai de chargement. Un classement de ces méthodes selon leur qualité de prévision a été proposé.

Mots-clés : essai SPT, pieu, foré, sable, essai de chargement, capacitè portante, banque de données.

\section{Comparative analysis of SPT based methods of design of single bored piles}

In geotechnical literature, several SPT-based methods of design of axial capacity of single bored piles in sand are available: These methods were mainly derived from empirical analyses of static vertical pile loading tests, Significant scatter is often noticed when comparing the predictions of these approaches Such an aspect was rarely elucidated by comparative studies assessing the predictive capabilities of these methods from the experimental evidences of pile loading tests.

The paper is aiming at contributing to evaluate some commonly used approaches in practice by the analysis of a database of loading tests of bored piles in sandy soils by comparing the predicted vertical limit loads to the ones experimentally interpreted. These methods were ranked with respect to their quality of prediction.

Kcy words ; SPT test, pile, bored, sand, pile loading test, bearing capacity, database. 


\section{NOTATIONS}

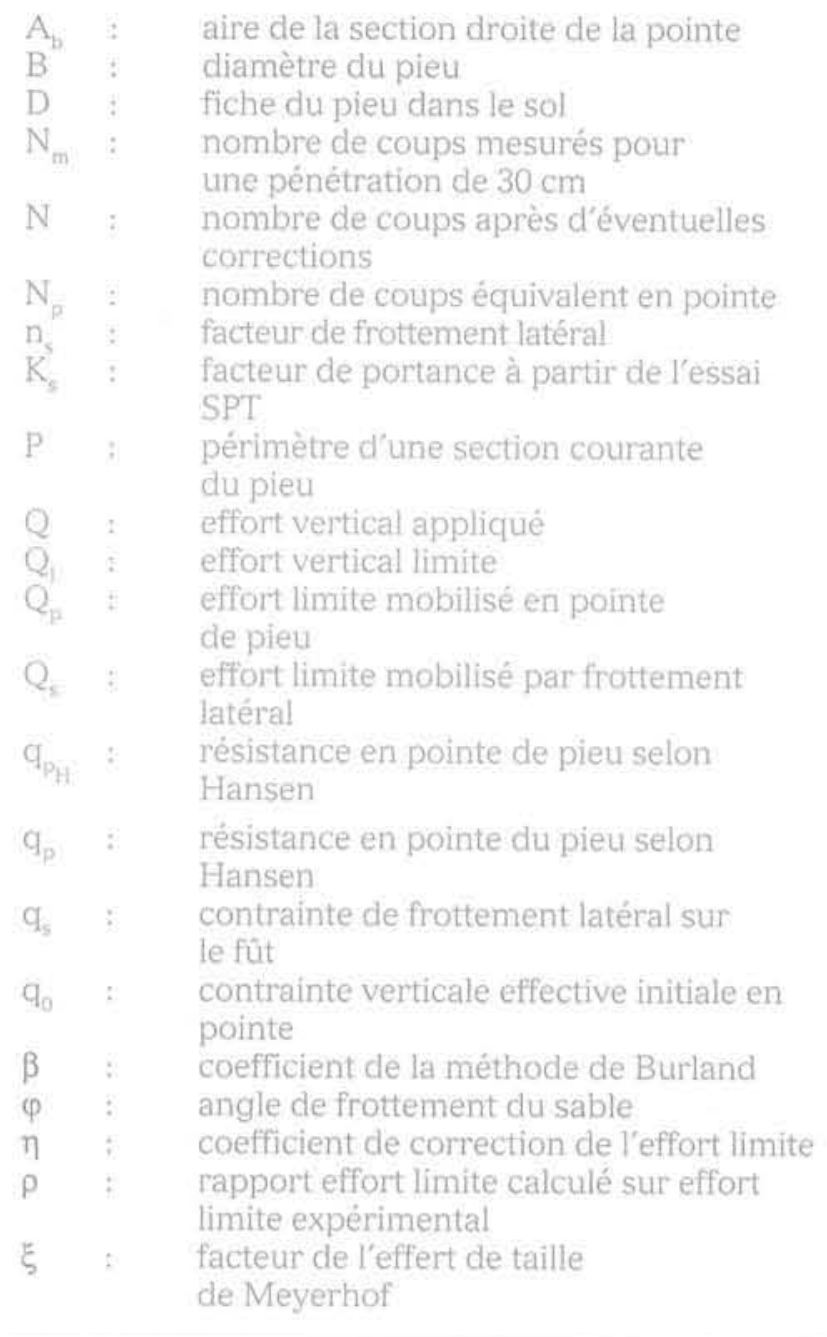

ment dû au caractère local de ces corrélations faites d'ailleurs dans un contexte géologique donné.

L'essai SPT, très bien adapté à la caractérisation des massifs sableux, s'est octroyé une place importante dans les projets géotechniques sur les continents américain et asiatique. Outre les avantages généraux des essais in situ, notamment la simplicité, la rapidité et le faible coût, cet essai permet d'extraire des échantillons remaniés des couches du terrain. Cependant, cet essai ne jouit pas en France de la même réputation que les essais pressiométrique et de pénétration statique, et il est ignoré dans les règles techniques françaises de conception et de calcul des fondations des ouvrages de génie civil (CCTG-93, fascicule 62, titre V). Le DTU 13.2, applicable aux fondations profondes des bâtiments, ne recommande aucune méthode de calcul et appelle à la prudence envers la diversité des formules de calcul.

Cette étude fait partie d'un programme de recherche sur le comportement des pieux forés aux Emirats arabes unis. Elle a pour objectif de présenter d'une part les méthodes courantes de calcul de la capacité portante des pieux à partir de l'essai SPT, et d'autre part les résultats d'une comparaison des prévisions faites à l'aide de ces méthodes avec les charges limites observées, à partir d'une banque de données de 50 essais de chargement de pieux forés dans 27 sites sableux légèrement limoneux aux Emirats arabes unis. Par la suite, un classement de ces méthodes, pour les sites étudiés, a été proposé selon un critère basé sur leur qualité de prévision.

\section{2}

\section{Présentation}

\section{des méthodes de calcul}

\section{1}

\section{Classification des méthodes}

Les méthodes d'évaluation de l'effort vertical limite peuvent être classées dans deux grandes catégories : - Les méthodes empiriques oủ la résistance en pointe $q_{0}$ est couramment supposée proportionnelle au nombre représentatif des coups $\mathrm{N}$ dans la zone mobilisée autour de la pointe, et telle que:

$$
\mathrm{q}_{\mathrm{p}}=\mathrm{K}_{\mathrm{s}} \cdot \mathrm{N}_{\mathrm{p}}
$$

$\mathrm{K}$ est le facteur de portance du SPT et a la dimension d'une contrainte.

La contrainte limite $q_{\text {s }}$ du frottement latéral le long du pieu est supposée proportionnelle au nombre $\mathrm{N}$ à l'interface sol/pieu, à la profondeur étudiée:

$$
q_{5}=n_{5} \cdot N
$$

$\mathrm{n}_{\mathrm{s}}$ est le facteur de frottement latéral et a la dimension d'une contrainte.

- Les méthodes semi-empiriques issues de l'adaptation de certaines méthodes classiques de capacité portante aux observations expérimentales des pieux. On cite à ce titre, la méthode de Hansen (1970) pour la résistance en pointe combinée à celle de Burland (1973) pour le frottement latéral.

L'effort vertical limite Q, sur un pieu circulaire de diamètre $\mathrm{B}$ et de fiche $\mathrm{D}$ est usuellement calculé à par- 
tir des termes de pointe $\mathrm{Q}$ et de frottement latéral $\mathrm{Q}$ supposés découplés, comme suit :

$$
Q_{i}=Q_{p}+Q_{s}=q_{p} \frac{\pi \cdot B^{2}}{4}+\pi \cdot B \cdot \int_{0}^{D} q_{s} \cdot d z
$$

\section{2}

\section{Méthodes empiriques}

\section{anting}

\section{Aoki et Velloso (1975)}

Aoki et Velloso ont montré, à partir de leurs études sur les pieux, que le facteur $\mathrm{K}$ dépend de la nature du sol et du mode d'installation du pieu. Les valeurs suggérées de $K_{s}$, résumées dans le tableau I, montrent que la rugosité d'interface sol/pointe n'intervient pas dans la résistance. Le nombre $N$ à introduire dans cette formule est la moyenne des trois valeurs de $\mathrm{N}$ les plus proches de la pointe. Le facteur $n$, du frottement latéral, résumé au tableau II, dépend de la nature du sol et du type de pieux et varie de 1 à $5 \mathrm{kPa}$.

\section{angas}

\section{Bazarra et Kurkur (1986)}

Bazaraa et Kurkur (1986) ont présenté une étude originale basée sur l'expérience d'essais de chargement des pieux en Égypte. Ils distinguent plusieurs catégories de pieux, comme le montre le tableau I. Le nombre $\mathrm{N}$ est défini identiquement à Van der Veen (1957), comme étant une moyenne arithmétique des valeurs de $\mathrm{N}$ sur une zone s'étalant de $3,75 \mathrm{~B}$ au-dessus de la pointe, à 1B au-dessous, sans toutefois dépasser une valeur moyenne maximale de 50 coups.

Les valeurs du facteur $n$, sont résumées au tableau II. Pour les sols pratiquement homogènes, les auteurs recommandent de prendre dans le calcul du frottement latéral q, une valeur moyenne du nombre $\mathrm{N}$ le long du pieu.

\section{Decourt (1982)}

Decourt (1982) a proposé une méthode d'évaluation de la résistance en pointe dans les sols, avec un facteur $\mathrm{K}$ récapitulé au tableau I pour les pieux forés, en béton préfabriqué, les pieux Franki ou les pieux Strauss. La valeur de $N_{\text {p }}$ représentative n'a pas été précisée. Le facteur de sécurité suggéré est de 4 pour la résistance en pointe.

Le frottement latéral dans les sables, limon et argiles est calculé comme suit :

$$
\mathrm{q}_{\mathrm{s}}=10 \times(\mathrm{N} / 3+1) \text { en } \mathrm{kPa}
$$

Le nombre $\mathrm{N}$ à introduire dans cette expression doit être entre 3 et 15 pour les pieux préfabriqués, pieux Franki et les pieux Strauss, et ne dépassant pas 50 pour les pieux forés. Le coefficient de sécurité suggéré est de 1,30 (Poulos, 1989 ; Bandini et Salgado, 1998).
TABLEAUI Valeurs du facteur de portance $\mathrm{K}_{\text {, }}$ dans les sables.

\begin{tabular}{|c|c|c|c|c|}
\hline \multirow[t]{10}{*}{$\mathrm{N}^{\mathrm{a}}$} & Méthode & \multicolumn{2}{|r|}{$\mathrm{K}_{\mathrm{n}}(\mathrm{kPa})$} & Remarques \\
\hline & \multirow{9}{*}{ Aoki-Velloso (1975) } & 286 & Sable & \multirow{3}{*}{$\begin{array}{l}\text { Pieux } \\
\text { forés }\end{array}$} \\
\hline & & 228 & $\begin{array}{l}\text { Sable } \\
\text { limoneux }\end{array}$ & \\
\hline & & 171 & Sable argileux & \\
\hline & & 571 & Sable & \\
\hline & & 457 & $\begin{array}{c}\text { Sable } \\
\text { limoneux }\end{array}$ & $\begin{array}{l}\text { Pleux } \\
\text { en béton }\end{array}$ \\
\hline & & 343 & $\begin{array}{l}\text { Sable } \\
\text { argileux }\end{array}$ & \\
\hline & & 571 & Sable & \\
\hline & & 457 & $\begin{array}{l}\text { Sable } \\
\text { limoneux }\end{array}$ & $\begin{array}{l}\text { Pieux } \\
\text { en acier }\end{array}$ \\
\hline & & 343 & Sable argileux & \\
\hline 2 & Bazaria-Kurkur (1986) & $\begin{array}{l}B \leq C \\
B>C \\
x B C\end{array}$ & $\begin{array}{l}0.5 \mathrm{~m}: \mathrm{K}=200 \\
0.5 \mathrm{~m}: \mathrm{K}=400 \\
B \text { en m) }\end{array}$ & $\begin{array}{c}\text { Pieux battus } \\
\text { ou injectés } \\
\text { sous haute } \\
\text { pression }\end{array}$ \\
\hline & & $\begin{array}{l}B \leq C \\
B>C \\
B(B\end{array}$ & $\begin{array}{l}0,5 m: K_{s}=135 \\
0,5 m: K_{s}=270 \times \\
\text { en } m \text { ) }\end{array}$ & Pieux forés \\
\hline 3 & Decourt (1982) & 400 & & Sable \\
\hline 4 & & 98.4 & & Sable \\
\hline & $\begin{array}{l}\text { Lopes-Laprovitera } \\
\text { (1988) }\end{array}$ & 87,0 & & $\begin{array}{c}\text { Sable } \\
\text { limoneux }\end{array}$ \\
\hline & & 87,0 & & $\begin{array}{c}\text { Sable } \\
\text { argileux }\end{array}$ \\
\hline 5 & Meyerhof (1976) & 120 & & Pieux forés \\
\hline & CGS (1985) & 400 & & Pieux battus \\
\hline 6 & Shiol-Fukil (1982) & 100 & & Pieux forés \\
\hline & & 300 & & Pieux battus \\
\hline 7 & Reese-O'Neill (1989) & $\begin{array}{l}0,5< \\
\mathrm{K}= \\
\mathrm{B} \geq\end{array}$ & $\begin{array}{l}\mathrm{B}<1,27 \mathrm{~m}: \\
60 \\
1,27 \mathrm{~m}: \mathrm{K}_{\mathrm{q}}=76 / \mathrm{B}\end{array}$ & Pieux fores. \\
\hline 8 & PHRI (1980) & 400 & & Pieux battus \\
\hline 0 & Bohert (4007) & 115 & & Pieux fores \\
\hline 3 & Hobert (f9y) & 190 & & Pieux battus \\
\hline 10 & Yamashita et al. (1987) & 150 & & $\begin{array}{l}\text { Pieux coulés } \\
\text { sur place } \\
q_{p}<7.5 \mathrm{MPa}\end{array}$ \\
\hline 11 & Martin ef al. (1987) & 450 & & $\begin{array}{l}\text { Pieux battus } \\
\text { avec pointe } \\
\text { fermée }\end{array}$ \\
\hline
\end{tabular}

Values of bearing capacity factor $\mathrm{K}$, for sands.

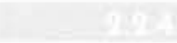 \\ Lopes et Laprovitera (1988)}

Lopes et Laprovitera ont étudié des essais de chargement des pieux forés et ont proposé de modifier la méthode d'Aoki et Velloso. Le nombre $\mathrm{N}_{n}$ est une moyenne arithmétique des valeurs de $\mathrm{N}$ entre $-B$ et $+B$ de part et d'autre de la pointe.

\section{rag.5. \\ Meyerhof (1983)-CGS (1985)}

Meyerhof a proposé une méthode dans laquelle le facteur de portance dans le sable dépend de l'élance- 
tABlEAU H Valeurs du facteur de frottement latéral $n_{\text {a }}$ dans les sables.

Values of skin friction $n$, for sands.

\begin{tabular}{|c|c|c|c|c|}
\hline $\mathrm{N}^{\mathrm{P}}$ & Méthode & \multicolumn{2}{|c|}{$n_{s}(\mathrm{kPa})$} & Remarques \\
\hline \multirow{9}{*}{1} & \multirow{9}{*}{ Aoki-Velloso (1975) } & 2.00 & Sable & \multirow{3}{*}{$\begin{array}{l}\text { Pieux } \\
\text { forés }\end{array}$} \\
\hline & & 2,28 & $\begin{array}{c}\text { Sable } \\
\text { limoneux }\end{array}$ & \\
\hline & & 2,57 & Sable argileux & \\
\hline & & 4,00 & Sable & \\
\hline & & 4,56 & $\begin{array}{l}\text { Sable } \\
\text { limoneux }\end{array}$ & $\begin{array}{l}\text { Pieux } \\
\text { en béton }\end{array}$ \\
\hline & & 5,14 & $\begin{array}{c}\text { Sable } \\
\text { argileux }\end{array}$ & \\
\hline & & 4,00 & Sable & \\
\hline & & 4,56 & $\begin{array}{l}\text { Sabie } \\
\text { limoneux }\end{array}$ & $\begin{array}{c}\text { Pieux } \\
\text { en acier }\end{array}$ \\
\hline & & 5,14 & Sable argileux & \\
\hline 2 & Bazaria-Kurkur (1986) & $\begin{array}{l}B \leq 0 \\
B>0 \\
X B(E\end{array}$ & $\begin{array}{l}, 5 m: n_{1}=2,20 \\
, 5 m: n_{2}=4,40 \\
B \text { en m) }\end{array}$ & $\begin{array}{c}\text { Pieux battus } \\
\text { ou injectés } \\
\text { sous haute } \\
\text { pression }\end{array}$ \\
\hline & & $\begin{array}{l}\mathrm{B} \leq 0, \\
\mathrm{~B}>0, \\
\mathrm{X} \mathrm{B} \text { iE }\end{array}$ & $\begin{array}{l}, 5 \mathrm{~m}: \mathrm{n}_{\mathrm{s}}=0,67 \\
, 5 \mathrm{~m}: \mathrm{n}_{\mathrm{s}}=1,34 \\
\text { Ben m) }\end{array}$ & Pieux forés \\
\hline 3 & Decourt (1982) & & $\begin{aligned}= & 10 \times(\mathrm{N} / 3+1) \\
& \mathrm{en} \mathrm{kPa}\end{aligned}$ & Sable \\
\hline 4 & & & 1.62 & Sable \\
\hline & $\begin{array}{c}\text { Lopes-Laprovitera } \\
\text { (1988) }\end{array}$ & & 1.94 & $\begin{array}{c}\text { Sable } \\
\text { limoneux }\end{array}$ \\
\hline & & & 3,06 & $\begin{array}{c}\text { Sable } \\
\text { argileux }\end{array}$ \\
\hline 5 & Meyerhof (1976) & & 1.00 & Pleux forés \\
\hline & CGS (1985) & & 2,00 & Pleux battus \\
\hline 6 & Shiot-Fukui (1082) & & 1,00 & Pieux forès \\
\hline to & 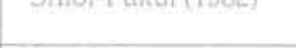 & & 2,00 & Pieux battus \\
\hline 7 & Wright-Reese (1979) & & 3,30 & Pieux forés \\
\hline 8 & PHRI (1980) & & 2,00 & Pieux battus \\
\hline 9 & Robert [1997] & & 1,90 & Pieux forés \\
\hline & & & 1.90 & Pieux battus \\
\hline
\end{tabular}

ment du pieu, de son mode de mise en place et de la présence ou non d'une nappe phréatique. Le tableau I résume les valeurs du facteur de portance pour des pieux ayant un élancement $\mathrm{D} / \mathrm{B}>10$. Le facteur de sécurité suggéré par Meyerhof est de 3 pour la résistance en pointe. Le nombre $\mathrm{N}$ à introduire dans le calcul est une moyenne arithmétíque des nombres $\mathrm{N}$ dans une zone entre $8 \mathrm{~B}$ au-dessus de la base et $3 \mathrm{~B}$ en dessous, corrigée par l'effet du poids des terres (Bowles, 1997).

En cas de terrain submergé par une nappe phréatique, il est recommandé de diviser ces facteurs par 2. Pour des pieux dont le diamètre varie entre 0,50 et $2 \mathrm{~m}$, Meyerhof propose de tenir compte d'un effet de taille sur la résistance en pointe, en la réduisant par un facteur $\zeta$ tel que :

$$
\zeta=\left(\frac{B+0,5}{2 . B}\right)^{n}
$$

$\mathrm{n}=1,2$ ou 3 selon que le sable est lâche, moyennement dense ou dense respectivement. B doit être exprimé en m (Bandini et Salgado, 1998). Pour des pieux battus dans le limon, Meyerhof propose d'adopter une valeur de $\mathrm{K}_{\mathrm{s}}$ égale aux deux tiers de celle du sable.

Meyerhof (1976), et par la suite le code canadien CFEM-85, ont suggéré de prendre $n_{8}$ égal à $2 \mathrm{kPa}$ pour les pieux battus dans les sables ou limons, et à $1 \mathrm{kPa}$ pour les pieux forés dans les sables. Cette méthode consiste à calculer un frottement latéral moyen le long du pieu, en introduisant dans le calcul une moyenne arithmétique de $\mathrm{N}$.

Selon Cassan, dans les sables submergés, il faut diviser ces facteurs par 2, sans préciser s'il y a lieu de corriger les valeurs de $\mathrm{N}$ sous la nappe d'eau par l'effet de nappe (Cassan, 1978).

\section{sas \\ Shiol et Fukui (1982)}

Cette méthode est couramment utilisée au Japon. Pour les pieux forés dans le sable, le facteur de portance $\mathrm{K}_{\mathrm{s}}$ est de $100 \mathrm{kPa}$. Pour les pieux battus, ce facteur dépend de l'élancement D/B, D étant la fiche du pieu dans la couche porteuse, et prend la forme suivante pour $\mathrm{D} / \mathrm{B}<5$ (Poulos, 1989) :

$$
\mathrm{K}_{s}=100 \times(1+0.4 \times \mathrm{D} / \mathrm{B}) \leq 300
$$

La valeur maximale de $\mathrm{K}_{\mathrm{s}}$ correspond en fait à un élancement plus grand que 5 dans la couche porteuse. Pour les pieux tubulaires ouverts en pointe et battus, le facteur de portance recommandé est :

$$
\mathrm{K}_{\mathrm{s}}=60 \times \mathrm{D} / \mathrm{B} \leq 300
$$

\section{Reese-O'Neill (1989)}

L'interprétation des essais de chargement des gros pieux forés dans le sable a conduit les auteurs à suggérer que le facteur de portance soit égal à $60 \mathrm{kPa}$ pour des diamètres entre $0.52 \mathrm{~m}$ et $1,20 \mathrm{~m}$ et des valeurs de $\mathrm{N}$ entre 5 et 60 . La valeur de $\mathrm{q}_{\mathrm{p}}$ doît être limitée à $4,5 \mathrm{MPa}$. Le nombre $\mathrm{N}$, représentatif est une moyenne arithmétique sur une zone de 2 diamétres sous la pointe du pieu. Pour des gros pieux avec $B \geq 1,27 \mathrm{~m}$, le facteur de portance est à calculer comme suit :

$\mathrm{K}_{\mathrm{s}}=76,2 / \mathrm{B}$ en $\mathrm{kPa}, \mathrm{B}$ est à exprimer en mètres

Wright et Reese (1979) ont recommandé de prendre n, égal à $3,3 \mathrm{kPa}$ pour les pieux forés dans le sable (Poulos, 1989).

\section{Than \\ Institut PHRI (1980)}

Le règlement japonais des ports TSPHF (Technical Standards for Ports and Harbors Facilities) recommande la formule suivante pour les pieux battus dans les sols sableux:

$$
Q_{1}=K_{s} \cdot N_{p} \cdot A_{b}+n_{s} \cdot N_{s} \cdot P \cdot D
$$

avec $K_{\mathrm{s}}=400 \mathrm{kPa}$ et $n_{\mathrm{s}}=2 \mathrm{kPa}$.

Le nombre $\mathrm{N}_{\mathrm{p}}$ est calculé comme suit :

$$
N_{p}=\left(N_{1}+N_{2}\right) / 2
$$

$N_{1}=\min (N$ à la pointe du pieu, moyenne des nombres $\mathrm{N}$ sur une zone de 2B sous la pointe) 
$\mathrm{N}_{2}=$ moyenne arithmétique des nombres $\mathrm{N}$ sur une zone de $10 \mathrm{~B}$ au-dessus de la pointe

Dans les sables fins ou des sables limoneux saturés et ayant des valeurs mesurées $\mathrm{N}_{\mathrm{m}}$ plus grandes que 15 , celles-ci doivent être corrigées par la formule de Terzaghi pour tenir compte de l'effet d'absorption de l'énergie de battage par l'eau interstitielle :

$$
N=15+\frac{N_{m}-15}{2}
$$

\section{Ditis: \\ Robert (1997)}

Suite à l'analyse d'une trentaine d'essais de chargement de pieux de différents types dans le sable, Robert a proposé des valeurs empiriques à $K$ et $n_{\text {. pour les }}$ pieux forés et battus, récapitulés aux tableaux 1 et 2 respectivement.

\section{9}

\section{Yamashita et al. (1987)}

Les auteurs ont proposé pour les pieux coulés sur place dans les sols pulvérulents de prendre $\mathrm{K}_{5}$ égal à $150 \mathrm{kPa}$, tout en limitant la résistance en pointe à 7,5 MPa (Poulos, 1989).

\section{Martin et al. (1987)}

Martin et al. ont proposé de prendre un facteur $\mathrm{K}$ égal à $450 \mathrm{kPa}$ pour les pieux battus dans le sable et mobilisant un déplacement important du sol, tels que les tubes fermés en pointe. Le nombre $N_{\text {n }}$ est une valeur moyenne dans la zone de rupture locale, l'étendue de cette dernière n'étant pas précisée (Poulos, 1989).

Le tableau I montre que les valeurs du facteur $\mathrm{K}$. dans le sable, proposées par les différentes méthodes, sont disparates et varient de 60 à $570 \mathrm{kPa}$. En outre, les valeurs du facteur $n$, varient selon le tableau 2, de 1,0 a $3,5 \mathrm{kPa}$, ce qui se traduit par un rapport des frottements latéraux prévus par ces méthodes, variant environ du simple au triple. A titre indicatif, à la base cies corrélations existant entre le pressiomètre et le SPT dans les formations sableuses assez homogènes, on peut esti-

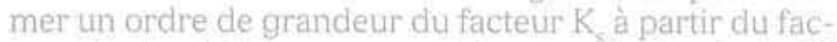
teur $K$ de résistance en pointe selon le fascicule 62 titre V. Selon Cassan (1978) et Bustamante (1989) le rapport $\mathrm{Pl} / \mathrm{N}$ se situe couramment dans les sables entre 50 et $70 \mathrm{kPa}$. Le facteur $\mathrm{K}$. varie de 1 à 1,2 pour les pieux forés dans les sables et graviers, ce qui donne pour $\mathrm{K}$. une plage de 50 à $85 \mathrm{kPa}$. Ces valeurs sont assez faibles par rapport à l'ensemble des méthodes exposées cidessus.

\section{5}

\section{Méthodes semi-empiriques}

Au cours de cette étude, la possibilité d'adaptation d'une méthode théorique aux résultats expérimentaux a été envisagée. La méthode généralisée de capacité portante d'une fondation enterrée due à Hansen (1970) a été adoptée pour l'évaluation de la résistance en pointe, comme suit :

$$
\mathrm{q}_{\mathrm{p}}{ }^{\mathrm{H}}=\mathrm{N}_{\mathrm{s} 1} \cdot \mathrm{s}_{\mathrm{q}} \cdot \mathrm{d}_{\mathrm{q}} \cdot \mathrm{q}_{\mathrm{o}}
$$

$q_{0}$ est la contrainte verticale effective initiale au niveau de la base du pieu; $N_{8}$ ' est le facteur de capacité portante d'une semelle continue selon la théorie de Hansen, fonction de l'angle de frottement $\varphi$ et corrigé par les facteurs de forme $\mathrm{s}_{\mathrm{f}}$ et de profondeur $\mathrm{d}_{\mathrm{f}}$ (Bowles, 1997). Ce dernier tend asymptotiquement vers une valeur constante pour les grandes fiches D, conformément au concept de profondeur critique pour la résistance en pointe.

Le frottement latéral est calculé avec la méthode $\beta$ due à Burland (1973), applicable aussi bien pour les sables que pour les argiles saturées. Il s'agit d'une méthode qui a été validée par des expériences de chargement des pieux (Bowles, 1997). Les contraintes à l'interface sol/füt du pieu sont supposées ètre à l'état $K_{r}$ et on décluit le frottement latéral par la relation:

$$
q_{s}=K_{0}, \sigma_{v 0}^{\prime} \cdot \operatorname{tg} \delta=\beta \cdot \sigma_{v 0}^{\prime}
$$

$\delta$ est l'angle de frottement de l'interface sol/fût dépendant de la rugosité de surface du fút. Il varie usuellement entre $15^{\circ}$ et $25^{\circ}$ et peut être pris en pratique égal à une fraction de l'angle de frottement interne $\varphi$ (Poulos et Davis 1980: Bowles 1997). La surface sol/fût a été supposée mi-rugueuse et l'angle $\delta$ a été pris égal à $0,75 \times \varphi$.

$\mathrm{K}_{0}$ est le coefficient des pressions des terres au repos, évalué usuellement comme suit :

$$
K_{0}=(1-\sin \varphi) \cdot(O C R)^{1 / 2}
$$

OCR est le rapport de surconsolidation du sable ou de l'argile. Ce facteur est à l'heure actuelle difficile à évaluer vu la difficulté d'extraction des échantillons sableux intacts reflétant thistoire des contraintes, mais il peut être estimé à partir des corrélations empiriques avec les essais in situ. Pour des raisons de simplicité, ce facteur a été pris dans cette étude égal à 1 (sable normalement consolidé).

L'abaque $\varphi-\mathrm{N}$ de Peck et al. (1973), couramment utilisé, a servi pour l'estimation de l'angle de frottement $\varphi$ requis. L'allure sensiblement linéaire de cet abaque pour $\mathrm{N}$ inférieur à 55 coups, peut être décrite par :

$$
\varphi^{\circ}=27,560+0,274 \times \mathrm{N}
$$

Conformément à Vesic (1970), le frottement latéra] q, n'augmente pas indéfiniment avee la profondeur. Au-delà d'une profondeur critique de 10 à 20 diamètres; $\mathrm{q}$, devient constant et il est alors recommandé de le limiter à $110 \mathrm{kN} / \mathrm{m}^{2}$ pour les sables très denses. Cette limitation, en accord avec le DTU-13.2 qui recommande un plafond de $120 \mathrm{kN} / \mathrm{m}^{2}$, a été adoptée dans cette étude.

L'effort vertical limite théorique, calculé à partir de l'équation 3 en introduisant les équations 14 et 15 , a été comparé à celui obtenu expérimentalement, ce qui a permis de définir un coefficient de correction $\eta$ égal au rapport entre le l'effort limite théorique et l'effort limite expérimental, Il a été constaté que ce coefficient varie peu avec l'élancement des pieux, mais augmente avec le nombre $\mathrm{N}_{p^{\prime}}$ comme l'illustre la figure 1. L'allure du nuage des points parait fluctuer raisonnablement autour d'une droite.

Il est à noter que la valeur représentative $\mathrm{N}_{\mathrm{p}}$ en pointe a été définie comme étant la moyenne des valeurs de $\mathrm{N}$ situées entre $2 \mathrm{~B}$ au-dessous de la pointe et $0,5 \mathrm{~B}$ au-dessus. Pour $87 \%$ des pieux étudiés, le coeffi- 


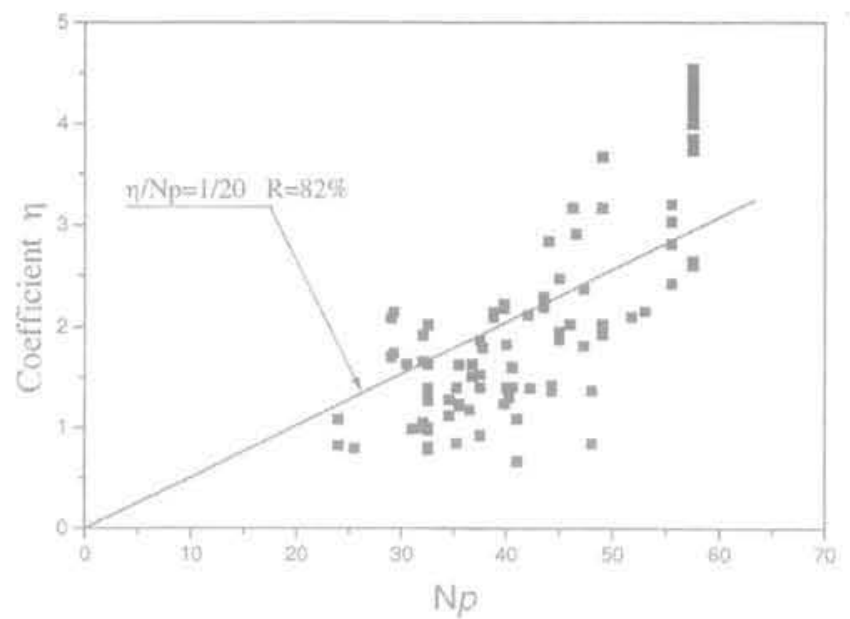

FIG, $t$ Variation du coefficient de correction $n$ avec $\mathrm{N}_{\mathrm{p}}$.

Correction factor $\eta$ versus the equivalent $\mathrm{N}$ value,

cient de variation $C_{\text {, de }} \mathrm{N}_{\mathrm{n}}$ est inférieur à $10 \%$, ce qui indique une faible dispersion des caractéristiques mécaniques du sol autour de la pointe. Pour le reste des pieux, soit $13 \%$, C peut atteindre $42 \%$. Le nombre $\mathrm{N}_{\text {. }}$ a été corrigé par l'effet de nappe, conformément aux recommandations de Terzaghi et Peck pour les sables saturés, à partir de l'équation (13).

\section{3}

\section{La banque des données d'essais de chargement}

\section{1}

\section{Contexte géotechnique}

Une banque de données a été construite à partir des sites représentatifs des Emirats arabes unis avec une taille initiale de 50 essais de chargement statique dans 27 sites, Cette taille a été jugée suffisante en phase préliminaire de ce projet de recherche. Les sites étudiès sont situés sur la cote du golfe arabe, et couvrent les principales capitales des émirats, notamment Dubai, Sharjah et Ajman.

Sur le plan géologique, une grande partie des dépôts superficiels est issue du Pléistocène et formés principalement du sable de dunes, du loss et des limons. La configuration typique des sols étudiés est constituée de couches de sable de granulométrie fine à moyenne, faiblement limoneux ou limoneux avec des traces de graves, de gypses, des fragments de coquilles ou de sable cimenté. Les échantillons étudiés en analyse granulométrique appartiennent souvent aux classes SW, SP et SM selon le système USCS, ce qui correspond respectivement aux sables bien gradués, mal gradués ou sables limoneux. Les couches sableuses sont caractérisées par un poids volumique sec de 14,5 à $17 \mathrm{kN} / \mathrm{m}^{3}$. Le niveau de la nappe phréatique est en général fluctuant et proche de la surface. Le nombre de coups $\mathrm{N}$ mesuré au niveau de la base des pieux étudiés varie entre 42 et 100 , ce qui correspond à une 16 plage de l'indice de densité $\mathrm{I}_{\text {di }}$ de 70 à $100 \%$, donc à des sables denses à très denses. La caractérisation des formations sableuses est menée avec l'essai de pénétration SPT qui, par sa simplicité et sa rapidité, est l'essai in situ le plus utilisé dans le pays. Il existe une diversité dans les équipements, mais l'appareil typique est doté d'un mouton de $63,5 \mathrm{~kg}$ avec une chute libre de $76 \mathrm{~cm}$. Le carottier échantillonneur a une longueur de $75 \mathrm{~mm}$ et un diamètre interne de $35 \mathrm{~mm}$. La figure 2 illustre une coupe typique de sondage SPT.

Les pieux retenus dans cette base de données ont des pointes encastrées dans un horizon sableux et sont loin de la couche rocheuse tendre formée essentiellement de grès. Les pieux encastrés dans le rocher ont été regroupés dans une autre banque de données et ont fait l'objet d'autres publications (Bouafia, 2002).

\section{2}

\section{Pieux d'essai}

Le tableau III résume les caractéristiques des pieux d'essais et celles des essais de chargement, Les pieux sont en général forés tubés ou forés à la boue, avec utilisation courante de la bentonite. L'instrumentation est souvent simple et comporte 4 comparateurs au 1/100 de mm pour la mesure du tassement $v_{0}$ en tête. Le chargement vertical Q est appliqué sous forme d'incréments par un vérin hydraulique en contact avec des

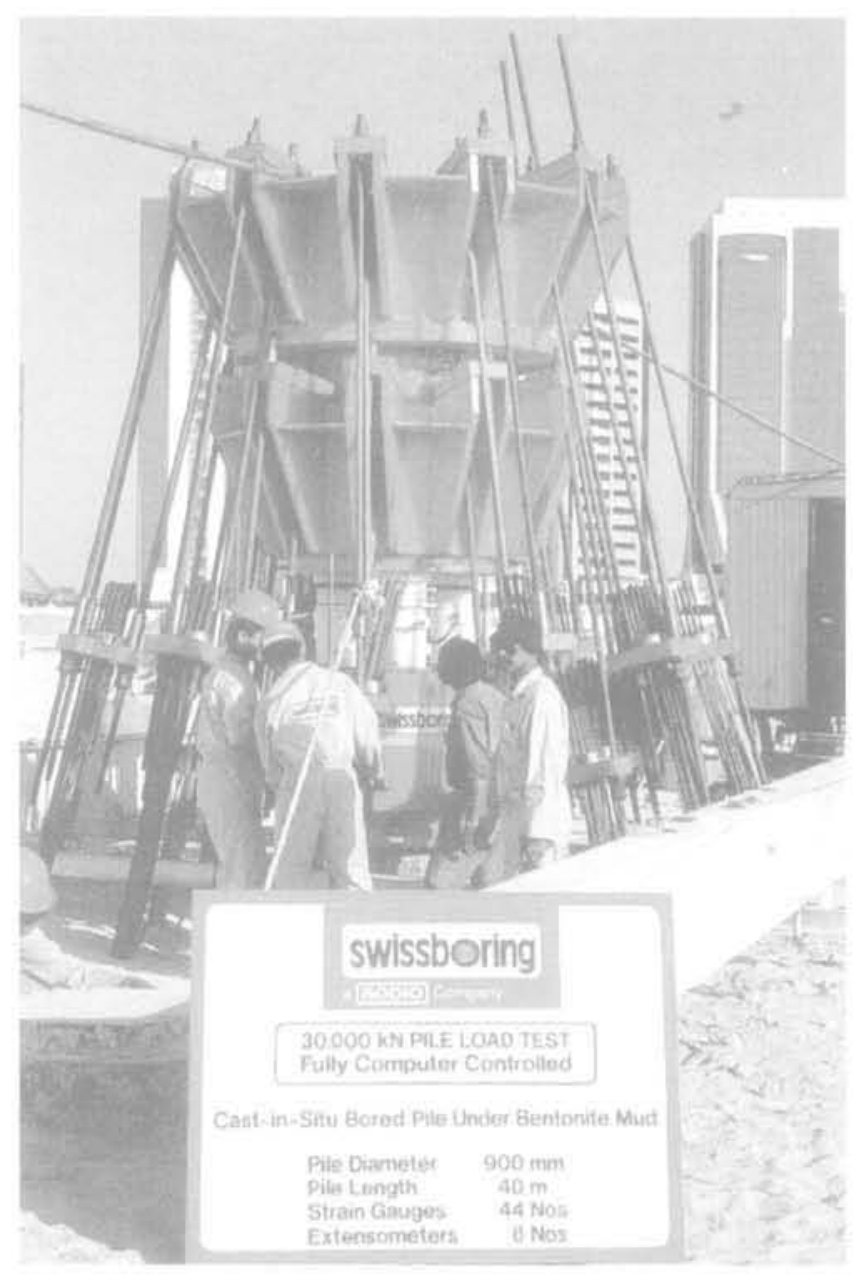

F1G. 3 Préparation d'un essai de chargement d'un pieu instrumenté.

Set-up of loading test of an instrumented pile. 


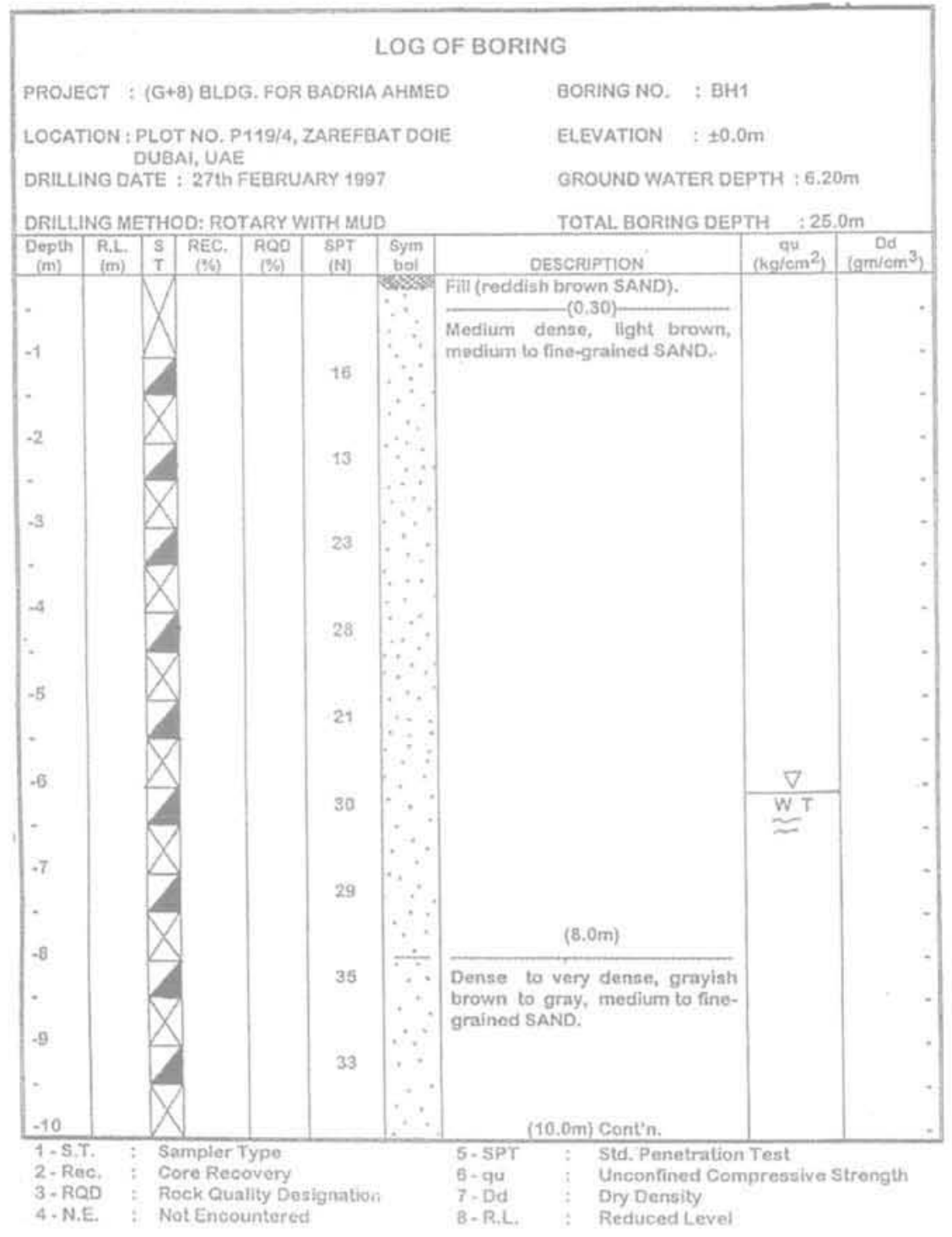

FIG. 2 Coupe typique de sondage SPT.

Typical SPT borehole log.

poutrelles, soit chargées par des contrepoids, soit en liaison avec des pieux d'ancrage. Dans certains projets, linstrumentation comporte des jauges de déformation et des extensometres amovibles, tels que le montre la figure 3.

TABLEAU III Caractéristiques des pieux et de l'essai de chargement.

Features of the test piles and the loading test.

\begin{tabular}{l|c}
\multicolumn{1}{c|}{ Caractéristique } & Valeur \\
\hline Élancement $\mathrm{D} / \mathrm{B}$ & $10-37$ \\
\hline Diamètre $\mathrm{B}(\mathrm{m})$ & $0,45-1,10$ \\
\hline $\begin{array}{l}\text { Résistance à la compression du béton } \\
\text { à 28 jours }(\mathrm{MPa})\end{array}$ & $20-40$ \\
\hline Effort vertical maximum appliqué $(\mathrm{kN})$ & $1200-9000$ \\
\hline Nombre de cycles de chargement & 2 \\
\hline Norme d'essai & ASTM D 1143-81
\end{tabular}

\section{3}

\section{Effort limite expérimental}

Il existe plusieurs critères d'analyse globale de la capacité portante des pieux non instrumentés, notamment ceux de Davisson, de Hansen, de Butler-Hoy, et le critère hyperbolique (ASCE, 1993). Le dernier critère a été adopté du fait que l'analyse d'ajustement des courbes de chargement $\mathrm{Q}-v_{0}$ montre que $92 \%$ des cas étudiés s'ajustent très bien à une courbe hyperbolique avec une asymptote horizontale pour les grands tassements. Le coefficient de régression obtenu est supérieur à $85 \%$, et la relation hyperbolique retenue est décrite comme suit :

$$
Q=\frac{v_{0}}{\frac{1}{\alpha}+\frac{v_{0}}{Q_{1}}}
$$

$Q$, correspond théoriquement à des tassements infinis et donc à l'asymptote horizontale de la courbe de chargement. Le coefficient $\alpha$ correspond à la pente initiale de la courbe de chargement. II est remarquable, 
comme l'illustre la figure 4, que l'effort limite ainsi interprété est pratiquement confondu avec celui conventionnellement défini comme correspondant à un tassement en tête égal à $10 \%$ du diamètre.

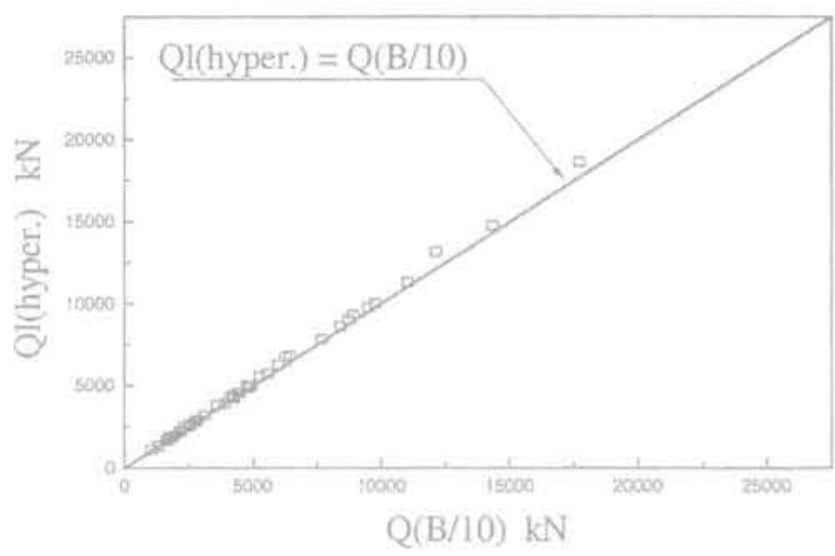

คG 4 Comparaison des efforts limites expérimental et conventionnel.

Comparison between experimental and conventional limit loads: mation est le pourcentage de cas où $p$ est inférieur à 1. Il est remarquable que la méthode 3 se distingue du reste des méthodes par une surestimation systématique de la capacité portante des pieux analysés. La méthode 1 présente également un taux de surestimation très fort égal à de $92 \%$. L'optimisme relatif de ces deux méthodes est probablement dû aux valeurs élevées proposées par les coefficients $K_{\text {s }}$ et $n$. Les méthodes 5 et 6 se sont avérées les plus pessimistes en sous estimant respectivement 95 et $83 \%$ des cas étudiés. D'autres études comparatives ont déjà montré le caractére pessimiste dé la méthode 5 (Turnbull et Kaufmann 1956; Mansur et Focht 1960 ; Bustamante et al., 1991).

La méthode 7 est caractérisée par des taux équilibrés de surestimation et de sous-estimation. Enfin, la figure 5 montre que le reste des méthodes, à savoir 9, 2 . 4 , et 10 ont une valeur moyenne du rapport $p$ de l'ordre de 0,95 (marge de 0,93 à 0,98 ) et le tableau IV indique que ces méthodes sous-estiment environ deux tiers des pieux étudiés.

On constate que le coefficient de variation $\mathrm{C}_{\mathrm{w}}$, qui est une mesure de la dispersion des résultats autour de la moyenne, est pratiquement le même pour toutes méthodes (marge de 30 à $35 \%$ ). Ces méthodes peuvent

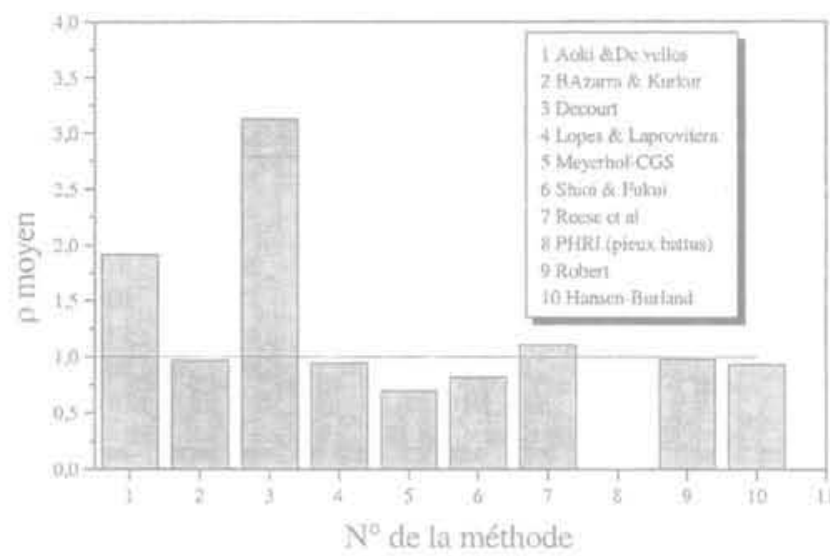

FiG.5 Histogramme de la valeur moyenne du coefficient $p$ des différentes méthodes. Histogram of the average value of $p$ for different methods. chaque méthode, la méthode de Hansen-Burland modifiée portant le numéro 10. Le taux de sous-esti-

\section{Analyse comparative des prévisions}

Les méthodes 1 à 9, résumées aux tableaux 1 et II ainsi que la méthode semi-empirique de Hansen-Burland corrigée ont été appliquées aux pieux d'essai. Dans la plupart des cas, la position du sondage SPT le plus proche du pieu d'essai n'est pas précisée. Pour tenir compte de la variabilité des caractéristiques du sol, le calcul a été fait au niveau de tous les sondages disponibles, ce qui a nécessité le traitement de 107 cas par chaque méthode. Une programmation sur microordinateur était donc nécessaire pour l'accomplissement de cette tâche.

On définit $\rho$ comme étant le rapport entre l'effort limite calculé et l'effort limite expérimental. Le ta-

tableAu IV Résultats de l'évaluation des méthodes de calcul. Results of assessment of the methods.

\begin{tabular}{|c|c|c|c|c|c|c|c|c|c|}
\hline Méthode & $\rho_{\text {ntiones }}$ & $P_{\text {nin }}$ & $P_{\text {max }}$ & Ecart-type: & $G(\%)$ & $\begin{array}{c}\text { Taux } \\
\text { de sous-estimation }(\%)\end{array}$ & $\begin{array}{l}\text { Taux } \\
\text { de surestimation }\end{array}$ & $\mathrm{P}(\%)$ & Rang \\
\hline 1 & 1.91 & 0.86 & 4.27 & 0,60 & 31,3 & 7,5 & 92,5 & 8.7 & 8 \\
\hline 2 & 0,97 & 0,35 & 1,95 & 0,31 & 31,8 & 58,0 & 42.0 & 48.0 & 2 \\
\hline 3 & 3,13 & 1,29 & 6,55 & 0,99 & 31.6 & 0,0 & 100,0 & 1,70 & 9 \\
\hline 4 & 0,95 & 0,37 & 2,40 & 0,32 & 33,2 & 68.2 & 31,7 & 46,7 & 3 \\
\hline 5 & 0,70 & 0,31 & 2,03 & 0,25 & 36,2 & 94,4 & 5,6 & 32.2 & 7 \\
\hline 6 & 0,82 & 0,36 & 1,86 & 0,27 & 31,6 & 83,2 & 16,8 & 45,4 & 4 \\
\hline 7 & 1.11 & 0,43 & 3,08 & 0.40 & 36,0 & 50,5 & 49,5 & 37.0 & 6 \\
\hline 9 & 0,98 & 0,44 & 2,56 & 0,34 & 35,1 & 66,3 & 33,7 & 43,7 & 5 \\
\hline 10 & 0.93 & 0,32 & 1,54 & 0,30 & 32,3 & 67,3 & 32,7 & 48.2 & 1 \\
\hline
\end{tabular}




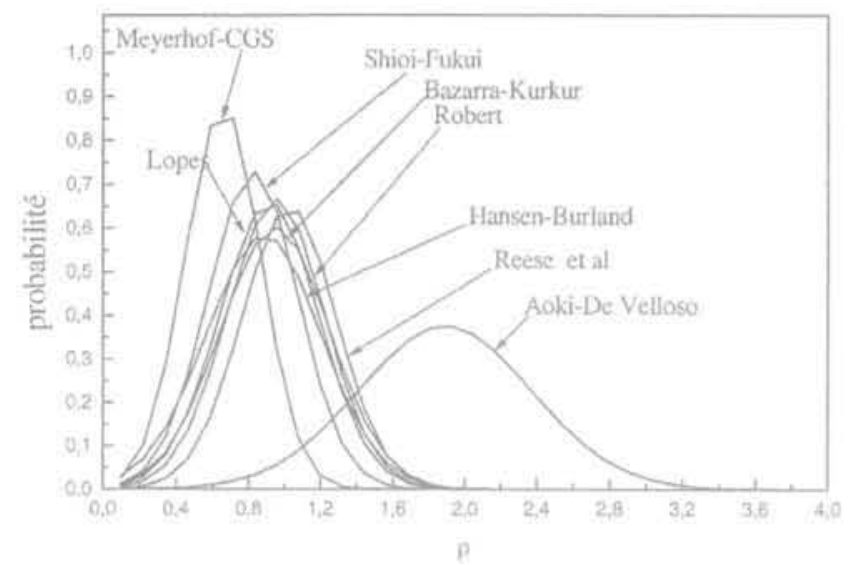

Fig.6 Distributions normales du rapport $\rho$ des différentes méthodes.

Gaussian distributions of the ratio $\rho$ for different methods.

être classées selon leur qualité de prédiction en tenant compte de la distribution des valeurs de $\rho$. En fait, l'incertitude dans les calculs de la capacité portante orientent plutôt vers la définition d'une plage admis. sible pour le rapport $\rho$, qui a été prise dans cette étude entre 0,8 et 1,20 . Les distributions statistiques de $\rho$ de chaque méthode s'apprêtent bien à un ajustement par la loi de Gauss comme le montre la figure 6. Ainsi, dans le tableau IV on a évalué pour chaque méthode, la probabilité $\mathrm{P}$ que $\rho$ se trouve dans la plage $0,8-1,2$ et on a classé les méthodes à partir de cette probabilité. Ainsi, les méthodes 10 (Hansen-Burland) et 2 (Bazarra-Kurkur) sont caractérisées par la plus forte probabilité, qui est de $48 \%$. Ce bon résultat pour la méthode 10 méthode théorique calée sur des observations expérimentales, et qui s'est avérée relativement meilleure aux autres méthodes, est encourageant au vu des différentes approximations faites au cours du processus de définition de cette approche. Des raffinements ultérieurs permettront d'améliorer encore sa qualité de prévision de la capacité portante.
Les méthodes de calcul exposées dans cette étude s'inscrivent dans une logique empirique à partir des observations des essais de chargement. La complexité du phénomène d'interaction sol/pieu au cours d'un chargement vertical et la diversité des paramètres géotechniques mis en jeu font que de telles approches ne peuvent prétendre à l'universalité. Il est ainsi attendu que les prévisions de capacité portante seraient divergentes, à cause du caractère local de ces observations, faites d'ailleurs dans un contexte géotechnique donné.

\section{5}

\section{Conclusion}

Une analyse comparative d'une dizaine de méthodes de calcul de la capacité des pieux forés dans le sable caractérisé par l'essai SPT a été menée à partir de l'exploitation d'une base de données de 50 essais de chargement statique de pieux dans des formations sableuses.

Après une présentation des différentes méthodes couramment utilisées en pratique, ainsi que celle utilisée pour exploiter la base de données, une méthodologie d'interprétation des données expérimentales a permis de comparer la méthode de Hansen-Burland aux mesures de la capacité portarite des pieux d'essais.

La qualité de prévision de la capacité portante par les différentes méthodes a été évaluée par comparaison directe des efforts calculés avec ceux obtenus expérimentalement. Un critère de classement de ces méthodes sur la base d'une plage admissible de la prévision de l'effort limite a été proposé. Il a été constaté que la méthode de Hansen-Burland permet une bonné prévision comparativement à d'autres méthodes courantes.

L'élargissement de la taille de la base de données, ainsi que l'amélioration de la qualité de prévision de la méthode de Hansen-Burland modifiée seront envisagés lors d'une étape suivante de ce projet de recherche

\section{Bibliographie}

Aoki, N., Velloso D. - « An approximate method to estimate the bearing capacity of piles v. Proceed. of the 5th Pan-American Conference on Soil Mechanics and Foundation engineering, vol. 1, Buenos Aires, $1975, \mathrm{p}, 367-376$

ASCE - "Bearing capacity of soils 1. Deep foundations, Technical Engineering and Design Guides, $n^{\circ} 7$. American Society of Civil Encineers, 1993. 142 p.

Bandini P., Salgado S. - "Methods of pile design based on CPT and SPT results n. Proceed. of the International Conference ISC'98 Geotechnical Site Characterization, Atlanta, A.A.Balkema, 1998. vol. 2, p. $967-976$

Bazarra A.R., Kurkur M.M. - «N-values used to predict settlements of piles in Egypt.3. Proceed. of ASCE: Use of in situ tests in geotechnical engineering. ASCE Geotech. Special Publication, vol. 6,1986, p. 462-474.

Bouafia A. - u Pile foundations bearing capacity-The UAE experience n. World of Engineering, Journal of the UAE society of engineers, $n^{*} 55$, Mav 2001.

Bouafia A. - "Analysis of axial capacity of socketed piles from 18 pile loading tests y. Proceed. of the 3 rd Intl. Seminar on Soil Mechanics and Geotechnical Engineering. Téhéran, Iran, ed. Iranian Geotechnical Society, 2002, 9 p.

Bowles J.E. - Foundation Analysis \& Design. McGraw-Hill Inc, 1997, 5th ed.. $1004 \mathrm{p}$.

Burland J.B. - " Shaft friction piles in clav-A simple fundamental approach n. Ground Engineering, vol. 6. $\mathrm{n}^{\circ}$ 3, p. 30-42.

Bustamante M. - « Aspect particulier de la portance des pieux v. Journées de mécanique des sols des LPC, Saint-Brieuc, 2022 juin 1989, 33 p.

Bustamante M., Frank R., Christoulas S: "Evvaluation de quelques méthodes de calcul des pieux forés $x$. Revue française de géotechnique, $\mathrm{n}^{*} 54,1991$, p. 39-52.

Cassan M. - Les essais in situ en mécanique des sols. Tome 2: "Applications et méthodés de calcul 2 , Eyrolles, Paris, 1978

Cassan M. - Les essais in situ en mécanique des sols. Tome 1 : «Réalisation et interprétation w, Evrolles, Paris, 1978

CGS - Canadian Foundation Engineering manuat CFEM. Canadian Geotechnical Society, c/o Bitech publishers Ltd, Vancouver, BC, 2nd ed., 1985.

Decourt L - «Prediction of the bearing capacity of piles based exclusively on N-Value of the SPT x. Proceed. of 2nd European Symposium on penetration testing, Amsterdam, vol. 1, 1982. p. 29-34.

Hansen J.B. - A revised and extended formula for Bearing Capacity. Danish Geotechnical Institute report, $n^{\circ} 28$, Copenhagen, 1970, $21 \mathrm{p}$.

Lopes R.F. Laprovitera H. - «On the prediction of the bearing capacity of bored piles from dinamic penetration tests n. Proceed, of Deep foundations on bored and auger piles conference 
BAP'88, Van Impe (ed.) 1988, P 537. 540

Meyerhof G.G. - " Bearing capacity and settlement of pile foundations $\mathrm{x}$. Journa of Geotech. Eng. ASCE, vol. 102, n 3 . 1976, p. 1-19.

Peck R.B., Hanson W.E., Thornburn T.H. -

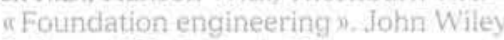
\& Sons, 1973, 2nd ed, 514 p.

PHRI - TSPHF : Technical Standards for Port \& Harbour Facilities in Japan. Chapter 4: w Bearing capacity of pile foundations n, 1980, p. 123-136.

Poulos H.G. Davis E.H. - «Pile foundation analysis and design w, Series in geotechnical Engineering, Lambe T.W. and
Whitman R.V. (eds), John Wiley \& Sons, 1980, $382 \mathrm{p}$

Poulos H.G - a Pile behaviour - Theory and application w. Géotechnique 39, 1989, n², p. 365.415.

Reese IC O'Neill. M.W - $\alpha$ New design method for drilled shafts from common soil and rock tests $x$. Proceed. of the congress Foundation Engineering-Current principles and practices, ASCE. ol.2. 1989, p. 1026-1039.

Robert Y, - " A few comments on pile design 1. Canaclian Geotechnical Joumal, col $34,1997, ?, 560-567$

Shiot Y., Fukui J. - « Application of N-value to design of foundations in Japan 3. Pro. ceed of the 2nd ESOPT, yol, 1, 1982 p. 159-164

Tornbull W i Kaufmann R. - w Discussion on the paper: Penetration tests and bearing capacity of piles in cohesiontess soils m, by Meverhof. Proceed. of ASCE. Journal of SMFE, vol, 82, ${ }^{\circ}$ SM.1, January 1956.

Van der Veen. Boersma L. - «Prévisons de la capacité portante d'un pieu à partir de fessai de pénétration statique $\pi$. Comptes rendus du 4 " congres international de Mécanique des Sols, Londres, 1957.

Vesic A.S. - a Tests on instrumented piles. Ogeechee river site $x$. Proceed, of the ASCE 96, SM.2, 1970, p. 561-584. 